\title{
Introduction to the special issue: value sensitive design: charting the next decade
}

\author{
Batya Friedman $^{1} \cdot$ Maaike Harbers $^{2} \cdot$ David G. Hendry $^{3}$ - $\cdot$ Jeroen van den Hoven ${ }^{4} \cdot$ Catholijn Jonker $^{5,6} \cdot$ Nick Logler $^{7}$
}

Accepted: 17 February 2021 / Published online: 29 April 2021

(c) The Author(s) 2021

\begin{abstract}
In this article, we introduce the Special Issue, Value Sensitive Design: Charting the Next Decade, which arose from a weeklong workshop hosted by Lorentz Center, Leiden, The Netherlands, November 14-18, 2016. Forty-one researchers and designers, ranging in seniority from doctoral students to full professors, from Australia, Europe, and North America, and representing a wide range of academic fields participated in the workshop. The first article in the special issue puts forward eight grand challenges for value sensitive design to help guide and shape the field. It is followed by 16 articles consisting of value sensitive design nuggets-short pieces of writing on a new idea, method, challenge, application, or other concept that engages some aspect of value sensitive design. The nuggets are grouped into three clusters: theory, method, and applications. Taken together the grand challenges and nuggets point the way forward for value sensitive design into the next decade and beyond.
\end{abstract}

Keywords Value sensitive design · Design research $\cdot$ Grand challenges $\cdot$ Design theory $\cdot$ Design methods $\cdot$ Applications

Value sensitive design (VSD), under development for over two decades, offers a theoretical and methodological framework for bringing human values into design processes (Friedman 2004; Van den Hoven 2013; Davis and Nathan 2014; Friedman and Hendry 2019). Computing and information technologies are increasingly being embedded into nearly all aspects of society and their impacts are pervasive, diffuse, and long-term.
To envision how the field of value sensitive design might be nurtured and developed over the next ten years a five-day workshop was developed: Value Sensitive Design: Charting the Next Decade, Lorentz Center, Leiden, The Netherlands, November 14-18, 2016, organized by the special issue editors. Forty-one researchers and designers, ranging in seniority from doctoral students to full professors, from Australia, Europe, and North America participated in

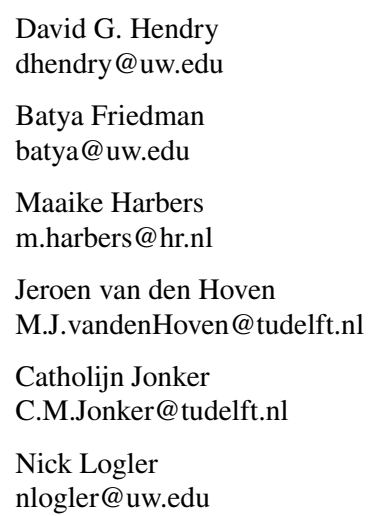

1 The Value Sensitive Design Lab, The Information School, University of Washington, Washington, USA

2 Research Center Creating 010, Rotterdam University of Applied Sciences, Rotterdam, The Netherlands

3 The Value Sensitive Design Lab, The Information School, University of Washington, Washington, USA

4 Delft Design for Values Institute, Delft University of Technology, Delft, The Netherlands

5 Interactive Intelligence Group, Fac. EEMCS, Delft University of Technology, Delft, The Netherlands

6 LIACS, Leiden University, Leiden, The Netherlands

7 The Value Sensitive Design Lab, The Information School, University of Washington, Washington, USA 
the workshop. ${ }^{1}$ The research disciplines represented at the workshop included civil and environmental engineering, computer science, design, digital media, ethics and technology, human-computer interaction, law and tech policy, library and information science, and management information systems.

This special issue of the Journal reports on this Lorentz workshop. The specific goals were (1) To identify a set of grand challenges to shape the next 10 years of work in value sensitive design; (2) To identify community resources that could be built to help the value sensitive design research community to flourish over the next decade; and (3) To build a value sensitive design research community comprised of young and senior scholars. These goals arose from a smaller one-day workshop the previous year at the Fifth Decennial Conference on Critical Alternatives, Aarhus, Denmark (Friedman et al. 2015).

The Lorentz workshop comprised a mix of varied formats, including distinguished conversations with leading researchers, workshopping papers, hands-on experiences with demonstrations of value sensitive design methods and pedagogical approaches, and many informal opportunities for intellectual exchange, including unconference sessions. The workshop also included time for interactive peer-review and writing sessions. In keeping with the aim of the workshop, participants worked on the identification of grand challenges. The workshop also gave participants an opportunity to write a "VSD nugget."

The first article of this special issue reports on eight grand challenges for value sensitive design. Grand challenges are open areas for new work that often benefit from collaborations among multiple researchers and research groups in order to make progress. Grand challenges should be compelling, motivating researchers to contribute to advancements in a field. A key goal of the Lorentz workshop was to identify a set of grand challenges that would help focus efforts of the value sensitive design community for the coming decade, bringing forward new issues, opportunities, aims, and aspirations.

The next 16 articles are value sensitive design nuggets. Put simply, a nugget is a short piece of writing on a new

\footnotetext{
1 The following individuals participated in the Lorentz Center Workshop: Tag Alshehri, Christian Bonnici, Alan Borning, Oliver Burmeister, Christian Detweiler, Batya Friedman, Christiane Grünloh, Maaike Harbers, Oliver Heger, Donal Heidenblad, David Hendry, Alina Huldtgren, Naomi Jacobs, Catholijn Jonker, Ian King, Marjolein Lanzing, Qinyu Li, Nick Logler, René Mahieu, Noëmi Manders-Huits, Jason Millar, David Miller, Lisa Nathan, Bryce Newell, Bjoern Niehaves, Anne Nigten, Nassim Parvin, Sarah Spiekermann, Luke Stark, Tjerk Timan, Ibo van de Poel, Jeroen van den Hoven, Peter van Waart, Aimee van Wynsberghe, Pieter Vermaas, Åke Walldius, Kari Watkins, Till Winkler, Volker Wulf, Daisy Yoo, and Annuska Zolyomi.
}

idea, method, challenge, application, or other concept, in this case engaging with value sensitive design. Perhaps the defining feature of a nugget is its focus on new work. It is an opportunity for an author to propose, focus on, and explore novel ideas in a short introductory format. With the work of a nugget in mind, this collection opens a window to some of the newest research and thinking in value sensitive design. The nuggets are grouped into three clusters: theory, method, and applications.

The first set of nuggets deal with theoretical considerations for value sensitive design. Till Winkler and Sarah Spiekermann review 20 years of value sensitive design case studies, and Naomi Jacobs and Alina Huldtgren argue for mid-level ethical theory to undergird value sensitive design. Both nuggets turn a self reflective and critical eye towards the current state of value sensitive design, while offering considerations for future projects. Ibo van de Poel introduces principles for designers to account for values changing over time, and Anne Weibert, Konstantin Aal, Markus Rohde and Volker Wulf introduce insights for designers to scale local experiences to global challenges. The theory nuggets conclude with David Hendry, Batya Friedman and Stephanie Ballard presenting value sensitive design as a formative theory.

The method nuggets propose new techniques to support the conceptual, empirical and technical investigations called for in value sensitive design. Åke Walldius' adaptation of concept mapping, Maaike Harbers' agent-based simulations, and Christiane Grünloh's use of technology frames, integrate established methods into value sensitive analyses. Annuska Zolyomi explores social networking sites as resources for value based research. Daisy Yoo's stakeholder tokens, Ian King's use of science fiction as a value scenario, and Oliver Heger, Bjoern Niehaves and Henrik Kampling's value declarations lay the groundwork for entirely new methodological approaches in value sensitive design.

The final set of nuggets emphasize application cases. Kari Watkins uses her experience creating tools to support public transit riders and urban planners to demonstrate how value sensitive design applies to challenges in transportation planning and decision making. Nick Logler's exploration of value tensions in the maker movement brings a value sensitive approach to an area attracting increased attention in research and popular culture. Qinyu Li, Anne Weibert, Marén Schorch, and Volker Wulf combine value sensitive and grounded design to propose e-portfolios as a tool for tracking and sharing projects. The set of application nuggets closes with Oliver Burmeister and David Krep's use of value sensitive methods to disrupt power relations in health studies.

For many years, value sensitive design has sought progress (not perfection) in the design of technologies that account for human values. This workshop represents one milestone toward advancing theory, method, and practice for value sensitive design. The grand challenges offer a compelling agenda for 
future research. The nugget collection provides a snapshot into the newest ideas, research and work in value sensitive design, and reveals the vibrant range of questions engaging value sensitive design scholars today. Whether you are a designer, researcher, engineer, ethicist, policy maker, or anyone working at the intersection of technology and society, we hope that you will be stimulated by this special issue.

Acknowledgements We would like to thank the Lorentz Center, Leiden, The Netherlands, for their generosity and gracious support of the 2016 workshop; they created a welcoming environment for the sustained intellectual conversations and discussions that have resulted in this special issue.

Open Access This article is licensed under a Creative Commons Attribution 4.0 International License, which permits use, sharing, adaptation, distribution and reproduction in any medium or format, as long as you give appropriate credit to the original author(s) and the source, provide a link to the Creative Commons licence, and indicate if changes were made. The images or other third party material in this article are included in the article's Creative Commons licence, unless indicated otherwise in a credit line to the material. If material is not included in the article's Creative Commons licence and your intended use is not permitted by statutory regulation or exceeds the permitted use, you will need to obtain permission directly from the copyright holder. To view a copy of this licence, visit http://creativecommons.org/licenses/by/4.0/.

\section{References}

Davis, J., \& Nathan, L. P. (2014). Value sensitive design: Applications, adaptations, and critiques. In J. van den Hoven, P. E. Vermaas, \& I. van de Poel (Eds.), Handbook of ethics, values, and technological design (pp. 1-26). Netherlands: Springer. https://doi. org/https://doi.org/10.1007/978-94-007-6994-6_3-1

Friedman, B. (2004). Value sensitive design. In W. S. Bainbridge (Ed.), Encyclopedia of human-computer interaction (pp. 769-774). Berkshire Publishing Group.

Friedman, B., \& Hendry, D. G. (2019). Value sensitive design: Shaping technology with moral imagination. MIT Press.

Friedman, B., Hendry, D. G., Huldtgren, A., Jonker, C., van den Hoven, J., \& van Wynsberghe, A. (2015). Charting the next decade for value sensitive design. Aarhus Series on Human Centered Computing, 1(1), 9-10.

van den Hoven, J. (2013). Value sensitive design and responsible innovation. In R. Owen, J. Bessant, \& M. Heintz (Eds.), Responsible innovation (pp. 75-83). John Wiley \& Sons.

Publisher's Note Springer Nature remains neutral with regard to jurisdictional claims in published maps and institutional affiliations. 